\title{
Contents Vol 38, 1915
}

\author{
Moiiaisschriff
}

fur

\section{Psychiatrie und Neuroiogie.}

\author{
Herausgegeben von \\ K. Bonhoeffer.
}

Bd. XXXVIII.

Mit zahlreiclien Abbildungen im Text und 2 Tafeln.

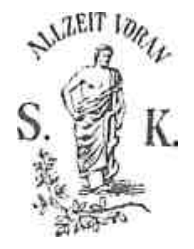

B E R L I N 1915 VERLAG VON S. KARGER KARLSTRASSE 15. 
Alle Rechte vorbehalten. 
- GedrucUt bei Imberg \& Lefson G. m. b. H. in Berlin SW 4.> 



\section{Inhalts -V erzeichnis.}

Original-Arbeiten.

Artom, G., Klinischer und patliologisch-anatoniischer Beitrag zum Studium des Hirnccliinococcus.

Bieling, R., Organische Erkrankungen mit hysterischer Pseudo-

demenz 268

Binswanger, 0., Hystero-somatische Krankheitserscheinungen

bei der Kriegshysterie

Bonhoeffer, K., Erfahrungen uber Epilepsie und Verwandtes

im Feldzuge.

Borchardt, Ludwig, Selbstverletzung am Schadel und Ge-

him

Brouw\&r, B. und H. J. Blauwkuip Uber das Zentralnervensystem bei pernizioser Anamie. (Hierzu Taf. I-II.) . . 2815 Forster, E., Der Krieg und die traumatischen Neuroscn. . . "el —, Ein Fall von Paralyse mit negativem Wassermann in Blut imd Liquor

Friedmann, M., Zur Auffassung der gehauften kleinen Anfalle.7ii Gennerich, W., Die Urs:v. hen von Tabes und Paralyse . . .341

Juliusburger, 0., Zur Kenntnis der Kriegsneurosen 305 Klaesi, J. und 0. Both, Uber einen Fall von Safrolvergiftung.235 Koppen, M., Uber das Gehirn eines Blindtieres Chrysochloris.201 .Kramer, F., Paralysis agitans-ahnliche Erkrankung . . . .171) Krueger, H., Uber lokalisierte Muskelatrophien bei Tabes dorsalis

Repond, A., Ein Fall von Katafconie nach Sonnenstich ... 08 - - tiber die Beziehunge'n zwischen Parasthesien und Halluzinationen besonders bei deliriosen Zustanden 21 (i Schroder, P., Traumatische Psychosen............................... 193 Schultz, J. H., Fiinf neurologisch bemerkenswerte Hirnschiisse319 Schivarz, E., Zwangsvorstellungen bei einem Hebephrenen .172 
Seelert, Hans, Uber Neurosen nach Unfallen mit besonderer

Beriioksichtigung von Erfahrungen im Kriege ....

Sittig, 0., Zur Pathogenese gewisser Symptome eklamptisoher

Psychosen................................................................................... 153

-, Wiederkehr des Patellarreflexes in eineni Falle von hoher Ruckenmarksdurchtrennung und die lokabsatorisohe Be-deutung des sogenannten „paradoxen Kniephanomens" . 257

Wasserjall, W.,MeningismTisimepileptischenDammerzustande 165

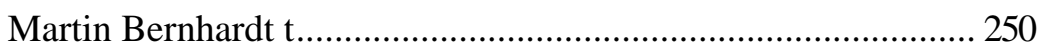

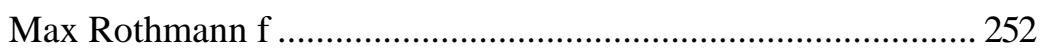

Buchanzeigen …........................................................... 128, 318- 